\title{
Investigación en Diseño, Arte y tecnología como base de resiliencia e innovación
}

\author{
Research in Design, Art and Technology as a basis for resilience and innovation
}

\author{
ANA GARCÍA-LÓPEZ (D) 0000-0003-3274-6651 \\ Universidad de Granada, Granada, España.
}

\section{Resumen}

El cambio de paradigma al que nos enfrentamos después de la crisis de la pandemia ha traído consigo una reflexión profunda acerca del modelo en que se desarrolla el progreso social y cómo es necesario marcar un tiempo nuevo en el que el diseño, la creatividad y la innovación transversal aplicada necesitarán ser trabajadas como disciplinas para su apropiación colectiva por la ciudadanía. 2021 se ha presentado como un año crucial en el que la economía creativa y los postulados de la nueva Bauhaus Europea marcan claramente un discurso en el que las naciones toman conciencia de la importancia de éstas como clave para la innovación, el desarrollo sostenible y, en fin, la recuperación económica. En este contexto, la investigación en arte y diseño aporta un nutriente de transferencia a la sociedad que se hace indispensable para el impulso de esa innovación transversal que ha de enfrentar un tiempo nuevo para la resiliencia y la sostenibilidad. Se presentan en este artículo las bases teóricas sobre las que se sustentan dos proyectos de investigación europeos en los que el diseño y el arte contemporáneo son puestos en relación directa a través de la inteligencia artificial, el machine learning y la economía circular con el fin de establecer conexiones entre artistas, artesanos, diseñadores, makers, productores y empresas de reciclaje a través de un modelo de gestión híbrido basado en las comunidades de conocimiento y el eco-diseño, integrando paradigmas de economía circular, economía creativa y economía colaborativa.

PALABRAS CLAVE: Diseño, tecnología, economía circular, economía creativa, resiliencia, IA.

CÓMO CITAR ESTE TRABAJO / HOW TO CITE THIS PAPER

García-López, A. (2021). Investigación en Diseño, Arte y tecnología como base de resiliencia e innovación. Umática. Revista sobre Creación y Análisis de la Imagen, 4.

https://doi.org/10.24310/Umatica.2021.v3i4.13134

Umática. 2021; 4. https://doi.org/10.24310/Umatica.2021.v3i4.13134
Artículo original

Original Article

Correspondencia/

Correspondence

Ana García López

agarcial@ugr.es

Financiación/Fundings Proyecto Europeo de I+D+i "RRREMAKER. Reuse Reduce Recycle Al-based platform for automated and scalable Maker culture in Circular economy". Proyecto $\mathrm{H}$-2020 Marie Curie Research and Innovation Staff Mobility (H2020-Marie SkXodowska-Curie Actions-RISE-2020, Ref.\#101008060)

Received: 31.07.2021 Accepted: 08.11.2021 


\title{
Research in Design, Art and Technology as a basis for resilience and innovation
}

\author{
ANA GARCÍA-LÓPEZ \\ Universidad de Granada, Granada, España.
}

\begin{abstract}
The paradigm shift we are facing after the pandemic crisis has brought with it a profound reflection on the model in which social progress is developed and how it is necessary to mark a new time in which design, creativity and applied transversal innovation will need to be worked on as disciplines for their collective appropriation by the citizenry. 2021 has been presented as a crucial year in which the creative economy and the postulates of the new Bauhaus clearly mark a discourse in which nations become aware of their importance as a key to innovation, sustainable development and, ultimately, economic recovery. In this context, research in art and design provides a nutrient of transfer to society that becomes indispensable for the impulse of this transversal innovation that has to face a new time for resilience and sustainability. This article presents the theoretical bases on which two European research projects are based, in which design and contemporary art are put in direct relation through artificial intelligence, machine learning and the circular economy in order to establish connections between artists, artisans, designers, makers, producers and recycling companies through a hybrid management model based on knowledge communities and eco-design, integrating paradigms of circular economy, creative economy and collaborative economy.
\end{abstract}

KEY WORDS: Design, Technology, Al, Circular Economy, Creative Economy, Resilience.

\section{Summary - Sumario}

1. Introducción

2. Paradigmas de la Investigación en Diseño: de la colaboración a los algoritmos, pasando por la sostenibilidad.

3. La artesanía contemporánea como caso de estudio: preliminares

4. I+D+i como nutriente de innovación en diseño y artesanía contemporánea

4.1. La estructura permanente: Cátedra de Innovación en Artesanía, Diseño y Arte Contemporáneo

4.2. Los proyectos de investigación: MakerArt y RRREMAKER

5. Conclusiones 


\section{Introducción}

En el año internacional de la Economía Creativa para el desarrollo sostenible según Naciones Unidas ${ }^{1}$ es pertinente llamar la atención sobre una forma de transformar la creatividad en resultados concretos que aporten crecimiento económico a través de la innovación, pensando sobre las relaciones en comunidad, promoviendo la diversidad cultural y el desarrollo humano sobre la base de la sostenibilidad, entendida ésta desde un punto de vista económico, social y medioambiental (Economía Circular). Los países son llamados a incrementar la participación y promoción de las industrias creativas, preservando y promoviendo al mismo tiempo el patrimonio y la diversidad cultural.

El contexto internacional es propicio, fundamentalmente en Europa (aunque no solo aquí) donde se ha establecido un modelo inspirado en la Bauhaus de Gropius Ilamada la Nueva Bauhaus Europea, anunciada por la presidenta Von der Leyen en su discurso sobre el estado de la Unión de 2020, que expresó la ambición de la UE de crear lugares, productos y formas de vida hermosos, sostenibles e inclusivos. La iniciativa promueve un nuevo estilo de vida en el que la sostenibilidad se ajusta al estilo, con lo que se contribuye a acelerar la transición ecológica en diversos sectores de nuestra economía y en nuestras sociedades, así como en otros ámbitos de nuestra vida cotidiana.

El modelo que Von der Leyen plantea para impulsar Europa y construir el mundo en el que queremos vivir está inspirado en la Bauhaus fundada por Walter Gropius en Weimar en 1919, una vanguardista escuela que basaba su filosofía del diseño en situar al ser humano como destinatario de la creación artística que debe tener un propósito y una utilidad para las personas, para la sociedad. Racionalidad, funcionalismo y heterodoxia estética en periodo de entreguerras en una corriente integradora del arte a la arquitectura.

1. Año Internacional de la Economía Creativa para el Desarrollo Sostenible (2021)

El 19 de diciembre de 2019 la Asamblea General de las Naciones Unidas en la Resolución 74/198 decide declarar el año 2021 Año Internacional de la Economía Creativa para el Desarrollo Sostenible.

La Asamblea General,

(...) Reconociendo que la economía creativa, conocida en varios países como la "economía naranja", entraña, entre otras cosas, actividades económicas basadas en el conocimiento y la interacción entre la creatividad humana y las ideas, el conocimiento y la tecnología, así como los valores culturales o el patrimonio artístico y cultural y otras expresiones creativas individuales o colectivas, (...)

(...) 1. Decide declarar el año 2021 Año Internacional de la Economía Creativa para el Desarrollo Sostenible;

2. Alienta a todos los Estados Miembros, a las organizaciones del sistema de las Naciones Unidas y a otras organizaciones internacionales y regionales, así como a la sociedad civil, el sector privado, las organizaciones no gubernamentales, los círculos académicos y las personas, a que celebren el Año Internacional de manera apropiada y de conformidad con las prioridades nacionales, a fin de crear conciencia, promover la cooperación y el establecimiento de redes, alentar el intercambio de mejores prácticas y experiencias, aumentar la capacidad de los recursos humanos, promover un entorno propicio a todos los niveles y hacer frente a los problemas de la economía creativa (...)

Umática. 2021; 4. https://doi.org/10.24310/Umatica.2021.v3i4.13134 
La Nueva Bauhaus de Von der Leyen pasa por la creación de un "espacio de co-creación en el que arquitectos, artistas, estudiantes, ingenieros y diseñadores trabajen juntos"2 conseguir generar una nueva transición económica que actualice los cimientos de la antigua Bauhaus, con sinergias entra las nuevas tecnologías y el cuidado del medio ambiente. Un modelo en el que Europa ha de liderar los estándares de lo digital poniendo el énfasis en la tecnología, en especial en la inteligencia artificial arbitrada por regulaciones donde el control sobre los datos personales no sea de las empresas y de la inversión en áreas rurales donde aún no llegan las conexiones rápidas de banda ancha.

La oportunidad está fundamentada en la iniciativa creativa e interdisciplinar que convoca un espacio de encuentro para rediseñar nuestras futuras formas de vida, situado en la encrucijada entre el arte, la cultura, la inclusión social, la ciencia y la tecnología. Esta iniciativa transita el sello del "Green Deal" ${ }^{\text {a }}$ nuestros lugares de vida y se engloba en el esfuerzo colectivo para imaginar y construir un futuro que sea sostenible, inclusivo y hermoso para los ciudadanos del mundo. "Tenemos que pensar la forma en la que vivimos (...). El Pacto Verde es un cambio sistémico que necesitará amplio apoyo y mucha imaginación y creatividad (...). La nueva Bauhaus Europea es un puente entre el mundo de la ciencia y la tecnología y el de las artes y la cultura"4.

Incluir la noción de lo hermoso, lo bello, en una de las más ambiciosas políticas que se han lanzado en el entorno europeo para la recuperación económica después de la pandemia no es baladí y tiene gran importancia, pues alude a la expresión de los espacios inclusivos y accesibles donde el diálogo entre diversas culturas, disciplinas, géneros y edades se convierta en una oportunidad para imaginar un lugar mejor para todos. También significa una economía más inclusiva, donde la riqueza se distribuye y los espacios son asequibles. Alude igualmente a encontrar soluciones sostenibles que creen un diálogo entre nuestro entorno construido y los ecosistemas del planeta. Significa realizar enfoques regenerativos inspirados en los ciclos naturales que reponen los recursos y protegen la biodiversidad. Hermoso significa, al fin, establecer experiencias enriquecedoras que respondan a las necesidades más allá de nuestra dimensión material, inspiradas en la creatividad, el arte y la cultura. Significa apreciar la diversidad como una oportunidad para aprender de los demás. La transformación post pandémica pasa, pues, por la innovación, la experimentación y la conexión, a través de tender puentes entre ciencia, tecnología, arte y cultura como oportunidad para transformar nuestras vidas a través de la co-creación. Es necesario poner de relieve el valor de la simplicidad, la funcionalidad y la circularidad de los materiales sin comprometer la necesidad de comodidad y atractivo en nuestra vida cotidiana.

2. Ver en https://ec.europa.eu/commission/presscorner/detail/en/SPEECH_20_1655

3. Pacto Verde Europeo. Ver en https://ec.europa.eu/info/strategy/priorities-2019-2024/ european-green-deal_es

4. Ver en https://europa.eu/new-european-bauhaus/index_en 


\section{Paradigmas de la Investigación en Diseño: de la colaboración a los algoritmos, pasando por la sostenibilidad.}

En este contexto el término co-diseño es la colaboración durante el proceso de diseño que se puede producir en diferentes etapas y fases: desde encuestas o entrevistas para saber la opinión de los clientes, la observación para conocer comportamientos con productos desarrollados, o con el "hacer", para buscar soluciones mediante la creatividad conjunta (Steen, et al., 2011). El diseño puede abarcar la organización, los servicios y los productos. Estos procesos colaborativos cambian por completo los procesos tradicionales. Así los diseñadores/as deben aprender a aceptar e integrar a socios, expertos e inexpertos, en el proceso de diseño (Sanders y Stappers, 2008), pero también es rompedor para la figura del artesano/a tradicional, que debe incorporar en el proceso a otros agentes e interactuar con ellos (González, 2020).

Las nuevas dinámicas sociales, culturales y económicas que comienzan a desarrollarse desde la aparición de Internet, sugieren que, al estar potencialmente todos los individuos conectados, podemos construir múltiples relaciones orientadas a la participación lateral y la agrupación colectiva como factor esencial (Briceño, 2017). Aparece el concepto de Diseño distribuido que aboga por un nuevo enfoque del diseño que utiliza la conectividad global para mover datos, en lugar de productos. El diseño distribuido es uno de los resultados de la intersección de dos tendencias globales: el movimiento Maker y la digitalización de la disciplina del diseño. Esta convergencia ha dado lugar al surgimiento de un nuevo mercado, en el que los individuos creativos tienen acceso a herramientas digitales que les permiten diseñar, producir y fabricar productos por sí mismos o conectarse fácilmente a una red global de colaboradores para llevar a cabo aspectos de este proceso con ellos. Llamamos a este proceso y al consiguiente mercado que está surgiendo de estas tendencias, Diseño Distribuido 5 .

Si vinculamos la investigación a la utilización de algoritmos en la nube, llegamos al Diseño Generativo con el que se pueden crear formas complejas e inspiradas en la naturaleza con impresionantes patrones generativos que proporcionan una exploración alternativa automatizada desde el principio del diseño. En los últimos años, los sistemas generativos presentes en la naturaleza se han convertido en una fuente de inspiración para arquitectos y diseñadores. Cada vez son más los diseñadores que se interesan en las formas y los patrones orgánicos, buscan formas complejas, irregulares y repetitivas e intentan imitar los algoritmos de la naturaleza. Las herramientas de diseño asistido por ordenador, y más específicamente las herramientas de diseño paramétrico generativo, permiten conseguir estos objetivos y obtener técnicas de modelado más flexibles y adaptables ${ }^{6}$. El modelado basado en algoritmos plantea unas soluciones al diseñador que aumenta la flexibilidad creativa y

5. https://distributeddesign.eu/about/

6. Ver en: https://discover.zds.com/es/e-book-algorithmic-generative-design-cloud?utm_medium=cpc\&tutm_source=google\&tutm_campaign=202101_glo_sea_es_op51508_labl_generic_dsg_ewe_bm- 
explora más alternativas proporcionando posibilidades de recopilar y gestionar varios objetos al mismo tiempo en la experiencia de modelado.

El diseño basado en algoritmos permite modelar geometrías realmente complejas que supondrían un reto para las herramientas de diseño manual. Este diseño generativo implica una filosofía de modelado paramétrico, lo que significa que el diseño se puede manipular mediante parámetros variables, de modo que el diseñador puede explorar una gama más amplia de posibilidades con rapidez y poco esfuerzo. El grado de control sobre los sistemas de modelado que ofrecen las distintas interfaces y software en el mercado, permite a los usuarios estándar de estas herramientas convertirse en fabricantes de herramientas.

Si vinculamos estas prácticas y realizamos un ejercicio de transposición al entorno de los materiales sostenibles, estaremos ante una de las aportaciones más interesantes que el diseño ha implementado pues "en el universo de la reutilización de materiales, no necesariamente el producto final puede ser sostenible con el medio ambiente por la composición de los materiales utilizados primarios o secundarios". El ecodiseño ataja los problemas de raíz ya que es la "metodología para el diseño de productos [...] en el que el medio ambiente se tuvo en cuenta durante el proceso de desarrollo del producto como un factor adicional a los que tradicionalmente se utilizaron para la toma de decisiones" (Hermida y Balboa, 2014: 87).

En julio de 2011 se publicó la norma internacional ISO 14006:2011 "Directrices para la incorporación del ecodiseño"7 que ha sido posteriormente revisada en $2020^{8}$, la primera norma internacional de gestión medioambiental que integró aspectos propios del diseño, evaluación del impacto en el ambiente de los productos diseñados y la gestión y tratamiento de dichos impactos dentro de una organización o empresa. En ella se asevera que "las organizaciones están reconociendo tanto la necesidad de reducir los impactos adversos sobre el medio ambiente de sus productos como la necesidad de incluir consideraciones ambientales en el diseño y desarrollo, aplicando el concepto de ciclo de vida. Este proceso se denomina generalmente 'ecodiseño'. Y añade "el ecodiseño se define en este documento como un enfoque sistemático, que considera los aspectos ambientales en el diseño y el desarrollo con el objetivo de reducir los impactos ambientales adversos a lo largo del ciclo de vida de un producto".

El ecodiseño debería aplicarse a los productos nuevos y a los ya existentes, incluyendo la modificación de los procesos según sea necesario en la fabricación de los productos. Entre las razones que se esgrimen para integrar el ecodiseño en el proceso de diseño y desarrollo y que deberían ser aplicados en el proceso de fabricación artesanal están:

a) la creciente preocupación por el daño al medio ambiente, por ejemplo, el cambio climático, el agotamiento de los recursos, la pérdida de la biodiversidad o la contaminación;

m\&utm_term=dsg-generativedesign\&utm_content=search\&tgclid=CjwKCAjw2bmLBhBREiwAZ6ugolww6uM_m8TuAzfrUV8xgetsZbAaFaBzzhLPandUbf6_TzBHQKzhuBoCVYMQAvD_BwE

7. Organización Internacional de Normalización - ISO. (2013). Directrices para la incorporación de ecodiseño ISO14006:2011. Recuperado el 01/10/2020 de http://www.iso.org/iso/catalogue_detail?csnumber=43241

8. ISO 14006:2020 Environmental management systems - Guidelines for incorporating ecodesign Recuperado de https://www.iso.org/standard/72644.html el o1/10/2020

Umática. 2021; 4. https://doi.org/10.24310/Umatica.2021.v3i4.13134 
b) el reconocimiento de las oportunidades comerciales relacionadas con la eficiencia de los recursos y la economía circular (por ejemplo, estrategias para permitir un menor uso del carbono y el agua, así como estrategias de prolongación de la vida útil de los productos que incluyan la reutilización, la reparación, el reacondicionamiento y la re-manufactura de los productos);

c) la filosofía del ciclo de vida facilita por un lado la identificación de los requisitos ambientales relacionados con los productos por los clientes y otros actores interesados externas e internas; y por otro lado evita impactos ambientales que se desplazan involuntariamente dentro del ciclo de vida. (García López, 2021)

\section{La artesanía contemporánea como caso de estudio: preliminares}

A partir de las líneas conceptuales expuestas que están sirviendo de hoja de ruta para la renovación y la resiliencia en Europa y que se apoyan en la creatividad, el diseño y la colaboración entre arte, ciencia y tecnología, la artesanía contemporánea se abre como un nicho poco explorado en tanto a su capacidad para recibir el nutriente que le aporta la investigación, el desarrollo y la innovación proveniente de las muchas disciplinas que la transitan.

Desde la artesanía tradicional a la contemporánea, cercana a los postulados de la filosofía Maker, el nuevo artesano hace suyo el "hecho a mano" y lo transforma en el "hecho a mano digital" es decir, aprovecha aquello que le es útil, como ha venido haciendo a lo largo de los tiempos, poniendo su creatividad y su sabiduría al servicio de esa nueva artesanía que avanza desde la tradición. Se pueden poner por ejemplo el nacimiento de los llamados Fab Labs o laboratorios de Fabricación Digital en los que se practica el diseño distribuido para la fabricación sostenible, donde se establecen procesos de fabricación a través de la conexión de comunidades de todo el mundo que comparten herramientas de fabricación a través de la cooperación y el trabajo en red. El escenario de fabricación en el que la estructura binaria regía entre fabricar 10.000 piezas o una a una ha dejado de existir, un camino en mitad se ha abierto paso a través la digitalización de la producción y los procesos. Desde que Neil Gershenfeld creara el primer Fab Lab a principios del año 2000 en el Center for Bits and Atoms del MIT — Massachusetts Institute of Technologysu crecimiento ha sido exponencial y su planteamiento se ha difundido por todo el planeta. Uno de los motivos de su éxito se debe a la alternativa que representan frente al modelo tradicional de diseño, fabricación, distribución y consumo de bienes, un sistema que desde hace tiempo ha demostrado su ineficacia frente al desempleo, los problemas medioambientales y las necesidades de los consumidores ${ }^{9}$. Este nuevo paradigma constituye la mejor venganza de los artesanos (García López, 2020).

Algunas de las estrategias o líneas argumentales para la innovación en la artesanía entroncan precisamente con la co-creación y la colaboración, ya que como hemos mencionado

9. Ver en https://www.experimenta.es/noticias/grafica-y-comunicacion/experimenta-72-fab-lab-un-cambio-de-paradigma/ (Acceso 12/01/2021).

Umática. 2021; 4. https://doi.org/10.24310/Umatica.2021.v3i4.13134 
anteriormente, el entorno creativo de los artesanos, diseñadores y artistas se deben desarrollar en colectivos controlados que puedan producir resultados óptimos. Esta idea se puso en práctica durante los primeros Encuentros ADA Artesanos+Diseñadores+Artistas que en 2018 se desarrolló en la Escuela Albayzín de Artesanía de Granada y la Facultad de Bellas Artes de la Universidad de Granada. Este encuentro entre lo profesional y lo académico inició una senda de colaboraciones que puso en relación a profesionales en activo del sector artesanal con los estudiantes de Diseño y Bellas Artes de la Universidad, estableciendo una simbiosis creativa entre ambos que perdurara en el tiempo y generara nuevas vías de desarrollo y promoción laboral. Este primer encuentro se enmarcó en los Días Europeos de la Artesanía ${ }^{10}$, evento de carácter europeo, de modo que se confirió un entorno internacional a dichos encuentros. La estructura del I Encuentro ADA permitió la interacción durante más de un mes de los jóvenes diseñadores y artistas con los artesanos en sus talleres, conociendo la práctica y tecnologías, y encontrando un espacio común de colaboración y simbiosis en las llamadas "Cápsulas de Innovación en Artesanía". La estructura de los I encuentros ADA incluía unas sesiones de buenas prácticas por expertos en transferencia del conocimiento y modelos de co-creación, desde el punto de vista legal, empresarial y de autoría y derechos de propiedad intelectual. Esto produjo un ambiente de trabajo estable en el que se desarrollaron una gran variedad de prototipos que fueron presentados en el entorno de la European Maker Week de $2018^{11}$, al que se invitó a una selección de responsables de tiendas y negocios vinculados a espacios emblemáticos y muy turísticos de la ciudad (Alhambra, Museos de ciencias, Museos arqueológicos, Centro Lorca, Centro Falla, etc) recibiendo una buena aceptación y el apoyo de estos centros para el desarrollo de algunos prototipos sobre ciertas líneas creativas presentadas por parte de los equipos de trabajo.

El Centro Albayzín y la Universidad de Granada a través de la Facultad de Bellas Artes, el MediaLab y la Oficina de Software Libre participaron en la European Maker Week de 2016 ${ }^{12}$ evento europeo donde la innovación tecnológica y la artesanía estuvieron presentes de forma conjunta en el que se presentaron ejemplos de buenas prácticas, de colaboración, prototipos y proyectos vinculados con el movimiento Maker.

La pandemia de 2020 constituyó una oportunidad para reflexionar acerca del sector, sobre la nueva realidad y el encaje en ella del sector artesanal. Así, se diseñó ADA 2020 como un espacio de reflexión para el impulso de la artesanía, con cuatro sesiones de webinars, entre junio y julio de 2020, bajo el lema "Estrategias para el impulso de la artesanía"13 que invitó a expertos en cuatro paneles para debatir, entre otros, sobre modelos de negocio, tecnología y diseño, comunicación y venta, ayudas, etc. Las Jornadas tuvieron una amplia acogida y pu-

10. https://www.diasdelaartesania.es/

11. https://medialab.ugr.es/noticias/granadamakerday/

12. https://medialab.ugr.es/proyectos/maker-day-granada/

13. https://medialab.ugr.es/noticias/proyecto-ada-artesania-diseno-arte/ y https://www.youtube.com/ watch?v=fEO8ML2aTVQ

Umática. 2021; 4. https://doi.org/10.24310/Umatica.2021.v3i4.13134 
sieron de manifiesto el interés y la necesidad de los sectores e instituciones implicados, en el desarrollo de medidas de impulso en tiempos de crisis.

Precisamente la pandemia ha enfrentado al sector con problemas que ya venía sufriendo desde años atrás y que tienen relación con la adaptación de formatos, la naturaleza y composición de los productos o los canales de transmisión a través de los canales de difusión y distribución. En un análisis hecho a partir de una encuesta nacional realizada por investigadores de la UGR en colaboración con el Centro Albayzín ${ }^{14}$ se detectaron graves carencias en cuanto a la utilización de canales de venta on-line, entre otros. Estas carencias son especialmente sensibles a situaciones de falta de movilidad y por tanto de turismo, pues la dependencia de la presencia física de compradores es total.

Otra de las estrategias que están llamadas a impulsar la artesanía contemporánea es la aplicación de tecnologías ligadas a ramas de la ingeniería como son la robótica, la inteligencia artificial, el machine learning, la ingeniería de materiales o el diseño generativo, que pueden aportar una gran riqueza de posibilidades a los artesanos. Junto con la implementación de estrategias basadas en las tres Rs (Reciclar, Reutilizar, Reducir) el artesano ha de ser capaz de navegar y orientarse en la ideación de nuevas formas, con nuevos materiales y procesos provenientes de la reutilización de materiales. Estas estrategias, lejos de usurpar su lugar como homo faber, ayudarán a los artesanos a avanzar hacia un entorno más favorable para construir un modo de vida basado en la riqueza de la producción artesanal de vanguardia, donde la contemporaneidad no esté reñida con la tradición, ni lo local con lo global (García López, 2020).

\section{I+D+i como nutriente de innovación en diseño y artesanía contemporánea}

La artesanía es, como hemos dejado explicado, un territorio en el que se ha implementado poco la acción que la I+D puede aplicar y, por tanto, es un acontecimiento extraño e innovador en símismo. Desde los muchos ámbitos del conocimiento que se desarrollan en el seno de las universidades y centros de investigación se puede aportar un gran nutriente de investigación al sector, ayudando a su impulso y renovación.

El binomio Universidad/Artesanía no es frecuente y sin embargo sobre este par concurren de modo transversal grandes áreas del conocimiento que aportarían estudio, análisis, propuestas de mejora y estrategias de avance en materiales, diseño, mercado, economía, legal, cultura o inclusión, por nombrar sólo algunos. Entonces, se plantean estrategias que parten precisamente del ámbito de la investigación y el desarrollo, abordando algunos casos de éxito, en la consecución de proyectos de investigación financiada a nivel nacional e internacional y que vinculan artesanía, digitalización, ingeniería, materiales, economía circular, marketing digital e inteligencia artificial.

14. Acceso al Informe sobre la situación del sector de la artesanía en España durante la emergencia sanitaria del covid-19: https://zenodo.org/record/4265075\#.YCUtwlوKjaU

Umática. 2021; 4. https://doi.org/10.24310/Umatica.2021.v3i4.13134 
4.1. La estructura permanente: Cátedra de Innovación en Artesanía, Diseño y Arte Contemporáneo

A partir de las necesidades detectadas en el sector de la artesanía se plantea crear una estructura permanente que, desde la universidad, pueda vincular a investigadores de todas las áreas para abordar problemas concretos del sector. La creación de la cátedra en 2021 es producto del convenio con el Centro de Referencia Nacional de Artesanía dependiente de la Junta de Andalucía (Servicio Andaluz de Empleo) ${ }^{15}$.

La importancia del establecimiento de esta cátedra radica en la gran cantidad de nutrientes de innovación que la Universidad aporta al sector de la artesanía, en transferencia del conocimiento y en I+D desde las artes, el diseño, el marketing, la economía circular, la ingeniería basada en nuevos materiales, la ingeniería informática y la inteligencia artificial, la comunicación, el derecho, la antropología, etc, entre los muchos ámbitos del conocimiento involucrados. Desde una gran universidad con casi 500 años de historia como la de Granada cobra especial sentido la búsqueda de líneas de investigación que tengan un reflejo real en la transferencia del conocimiento en el entorno de la artesanía, máxime si tenemos en cuenta que la UGR es una de las que cuenta con mayor patrimonio cultural propio en el espectro de universidades españolas, con el expreso mandato de su salvaguarda.

La cátedra actúa de observatorio de la artesanía tradicional y plantea su puesta al día contemporánea a través de la innovación. Entre sus objetivos está fomentar líneas de investigación y proyectos que, a través del análisis de la evolución de las bases científicas y tecnológicas relacionadas con los procesos de formación o con el sector de referencia, aporte soluciones prácticas a partir de la transferencia entre la universidad y la empresa. Además, la implementación de acciones formativas permite a los profesionales participar en programas e iniciativas junto con los investigadores académicos para mejorar aspectos tales como la capacitación profesional de los jóvenes y la puesta al día de los veteranos en cuestiones tales como la transición a planteamientos de economía circular y sostenibilidad en productos y procesos, la internacionalización, el marketing digital y la distribución y difusión en redes.

En este contexto, esta cátedra nace con la intención de ser una incubadora de proyectos en la que investigadores de todos los ámbitos del saber aporten y nutran de corpus teórico y práctico a este sector, que generen nuevo conocimiento y enfoques innovadores para el desarrollo de modelos que puedan ser transferidos al tejido empresarial, sobre todo en Andalucía, pero que sean exportables a cualquier territorio. Bajo la estructura de la cátedra, la UGR y el CRN asumen el desafío de establecer una simbiosis productiva que abarca una enorme cantidad de líneas de investigación (desde la robótica a la Inteligencia Artificial, desde el eco-diseño al marketing emocional y desde las técnicas de mercado a la transición a la economía circular). El reto es amplio y el trabajo emocionante. La cátedra, que ha comenzado su andadura en 2021, tiene un enorme reto ante sí para, empujar a la artesanía a la vanguardia desde la tradición.

15. Ver noticia en la web de la UGR: https://canal.ugr.es/noticia/la-ugr-y-el-servicio-andaluz-de-empleo-crean-la-catedra-de-innovacion-en-artesania-diseno-y-arte-contemporaneo/

Umática. 2021; 4. https://doi.org/10.24310/Umatica.2021.v3i4.13134 
Un apoyo importante de esta estructura son los proyectos de investigación que son la base de la generación de nuevo conocimiento para ser transferido al tejido productivo, en este caso a los talleres artesanales y laboratorios de creación digital. A partir de las necesidades detectadas, se plantearon sendos proyectos, uno precursor de otro, en cuyas bases está la conexión del artesano tradicional con el artesano contemporáneo a través de la figura del eco-diseñador y con la asistencia de tecnologías de última generación. En los siguientes apartados vamos a describir los dos proyectos que vinculado los conceptos que se han vertido hasta ahora en estas páginas.

4.2. Los proyectos de investigación: MakerArt y RRREMAKER

A partir de las Muestras de Arte Efímero Spora y la Bienal del Milenio: Arte Contemporáneo y Patrimonio celebradas en granada en 2008, 2009 y 2011 se plantean las primeras experiencias de vincular artistas y diseñadores con procedimientos artesanos que se introducen en la obra de arte confiriendo una nueva vía de comunicación hacia la sociedad del patrimonio cultural. Fruto de esas experiencias nace el proyecto de I+D+i titulado "Comunicación y patrimonio a través del Arte contemporáneo. Transferencias culturales entre Arte, diseño y artesanía" financiado por el Campus de Excelencia Internacional de la Universidad de Granada, donde se catalogaron artesanías tradicionales y contemporáneas, y diseñadores y artistas actuales, que utilizan alguna destreza artesanal para la producción de sus piezas, en las ciudades de Tetuán (Marruecos) y Granada (España), por compartir una historia común.

De aquel proyecto se detectan ciertas carencias en cuanto a los procesos de digitalización del sector por lo que se plantea el proyecto MakerArt propuesta para la transformación digital de la industria cultural relacionada con la artesanía, a través del diseño, los procesos colaborativos y la cultura Maker que fue financiado por fondos FEDER dentro del reto "Economía y Sociedad Digital / Sociedades inclusivas, innovadoras y reflexivas" a través de convocatoria de la Junta de Andalucía en la convocatoria de 2018. Comenzó el 1 de enero de 2020 y estará activo hasta mediados de 2022. MakerArt plantea la creación de una plataforma de intercambio que es punto de encuentro de comunidades de productores de contenidos, donde se incluyen artesanos tradicionales, como los que empiezan a integrar tecnologías en su trabajo o los que realizan labores de producción y diseño con medios digitales. La plataforma, activa en http://makerart.es plantea oportunidades de colaboración a partir de la puesta en común de diseños y metodologías de producción que incorporen tecnologías maker, prácticas de comunicación y gestión de conocimiento y modelos de negocio que aprovechen las posibilidades del entorno digital. Dentro de la puesta en común de diseños y metodologías de producción se considera la implementación de un canal directo de intercambio de archivos de diseños entre agentes procedentes de cualquier parte del mundo. Entre otras múltiples posibilidades, por ejemplo, el artesano puede crear el objeto artesanal con técnicas propias de su oficio a partir del prototipo producido mediante impresión aditiva u otros métodos de reproducción digital. Así, a través de la colaboración y mediante el intercambio de prototipos en red, se pretende optimizar la producción y actualizar los diseños,
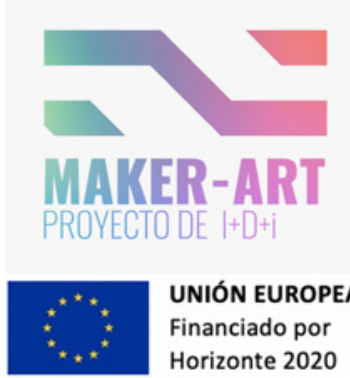

UNIÓN EUROPEA

Financiado por Horizonte 2020

Proyecto de $1+d+i$ "MAKER

ART: propuesta para la transformación digital de la industria cultural relacionada con la artesanía, a través del diseño, los procesos colaborativos y la cultura Maker", PROGRAMA OPERATIVO FEDER ANDALUCÍA 2014-2020. Ref.: B-HUM-407-UGR18, Reto "Economía y Sociedad Digital / Sociedades inclusivas, innovadoras y reflexivas" 01/01/2020 a 31/06/2022. IP: Dra. Ana García López. CoIP: Dr. Esteban Romero Frías. web: www.makerart.es 
produciendo un impacto en la modernización del sector, con repercusión en la industria cultural y turística, ofreciendo oportunidades de innovación social y emprendimiento para las nuevas generaciones.

Se han acometido las acciones de mapeo y análisis previstas para adquirir un conocimiento profundo de la situación actual del sector artesanal en nuestra comunidad, de modo que se puedan generar mecanismos que mitiguen las amenazas a las que se enfrenta; desde ese conocimiento se plantea favorecer el procomún utilizando la filosofía y el movimiento Maker, creando una estructura que potencie la co-creación por medio de las nuevas tecnologías. El resultado debe producir beneficios que redunden en la generación de productos más competitivos y de mayor valor, que diferencien el sector artesanal de nuestra comunidad frente a otras a través de la innovación.

El proyecto, además de este objetivo principal, plantea una serie de acciones para contribuir a la modernización del sector: propuestas de líneas de investigación vinculadas y acciones de formación en competencias digitales, como la implementación de tecnologías aditivas (impresión 3D), emprendimiento y formulación de nuevos negocios basados en la economía y producción colaborativa digital, para contribuir a la modernización del sector.

Las sinergias con otras instituciones existentes han sido clave para tejer la red del conocimiento. Así, a nivel nacional se agregó a la EOI-FUNDESARTE que es, desde 1981, la organización de referencia en la promoción y el desarrollo de las empresas artesanas españolas con el mandato de poner en valor y comunicar la calidad del producto artesano y la sostenibilidad de sus procesos de elaboración. Igualmente, importante es, a un nivel autonómico, la vinculación de las facultades de Bellas Artes de la comunidad o Escuelas de Arte. En lo local, indispensable ha sido la agregación del Centro Albayzín - nombrado Centro de Referencia Nacional de Artesanía en 2020 - o estructuras existentes en la Universidad de Granada como los laboratorios de fabricación (FabLabs), que ofrecen posibilidades reales de creación, investigación, educación y difusión de los resultados, además de la creación de nuevas líneas de trabajo.

Ya en 2018 cuando se formuló este proyecto, se adelantaba que, como resultado de estas acciones, se crearía una estructura estable desde la Universidad de Granada a través de una Cátedra de artesanía y diseño que aglutinara a los agentes implicados y catalizara las acciones descritas, daría continuidad al proyecto asumiendo la necesaria labor de salvaguarda, innovación y sensibilización de la artesanía tradicional y contemporánea hacia la sociedad. La cátedra, como hemos comentado, es una realidad y comienza su andadura en 2021. Pero hay que mencionar que es deudora de la intensa actividad que se desplegó a partir de la creación de grupos de trabajo creados en Medialab UGR (Vinculado al Vicerrectorado de Investigación y Transferencia de la UGR) bajo la propuesta Facultad Cero ${ }^{16}$, una propuesta creada en 2018 para repensar la Universidad desde una vocación abierta, global e innovadora, partiendo de la pregunta: "¿cómo diseñarías la Universidad si partiéramos de cero?". Nuestra propuesta (grupo de trabajo multidisciplinar creado para la ocasión) fue la creación

16. https://facultadcero.org/acerca-de/ 
de UGR ArtLab ${ }^{17}$, un laboratorio de creación e innovación para la artesanía, que dio paso a la estructura de Cátedra.

El germen formulado para MakerArt fue acicate para hacer crecer la idea hacia un consorcio internacional, intersectorial e interdisciplinar a través de la estructura del programa RISE (Research and Innovation Staff Exchange) que se apoya sobre la idea de intercambio entre necesidad (de las pequeñas y medianas empresas de Europa) y el potencial de investigación (de los grandes centros de investigación y las universidades). Dentro de este programa de intercambio de investigadores entre industria y academia, el proyecto "RRREMAKER: Reuse Reduce Recycle Al-based platform for automated and scalable Maker culture in Circular economy" (2021-2025) ha logrado la mejor calificación en su panel (Economía) para obtener uno de los dos proyectos financiados en dicho panel del programa Marie SkXodowska-Curie dentro del recién extinto Horizon2020. Durante cuatro años desde 2021, investigadores de 3 universidades y 7 empresas europeas y extracomunitarias planean para la consecución del objetivo principal de RRREMAKER que no es otro que desarrollar una plataforma maker basada en inteligencia artificial (IA) para el diseño y la producción de productos artesanales, de prototipado rápido y reacondicionados, a partir de la disponibilidad de bienes usados y residuos reciclables recogidos. Para ello, conectará entre sí a fabricantes digitales y artesanos tradicionales, diseñadores/empresas creativas y empresas verdes, estableciendo un nuevo modelo de gestión híbrido basado en las comunidades de conocimiento, el ecodiseño y la democratización de la invención, integrando la economía naranja, la economía colaborativa y la economía circular (https://www.rrremaker.com/).

RRREMAKER tiene un enfoque altamente disruptivo, porque además de ser una base sobre la que las diferentes áreas implicadas pueden compartir información, la propia plataforma computacional inteligente que se desarrolle será un actor principal en el proceso creativo, al integrar algoritmos de vanguardia de diseño generativo, que van a predecir y ofrecer al artesano un amplio espectro de estructuras, formas, materiales, colores, ornamentos, etc, basados en parámetros de diseño tradicional artesanal e innovador y materiales de reciclaje. La plataforma también se va a convertir en un "punto de clasificación" para compartir información entre los recolectores de materiales reciclables y los ecodiseñadores/fabricantes ecológicos y los artesanos, conectándolos entre sí. Además, a partir de la información de ventas archivada, la plataforma va a ser capaz de generar mapas de características de los productos ya comercializados y proponer automáticamente a los fabricantes cambios para hacerlos innovadores y más atractivos.

A partir de este proyecto de l+D+i europeo se vincula la artesanía con la economía circular y la ingeniería en un entorno que aporta además la internacionalización y la intersectorialidad, pues la participación de las PYMES europeas procura un ecosistema en el que la academia y la industria intercambian investigadores y conocimiento para aportar un beneficio social y transferible al sector de la artesanía.

17. https://facultadcero.org/2018/05/15/propuesta-laboratorio-art-lab/

Umática. 2021; 4. https://doi.org/10.24310/Umatica.2021.v3i4.13134

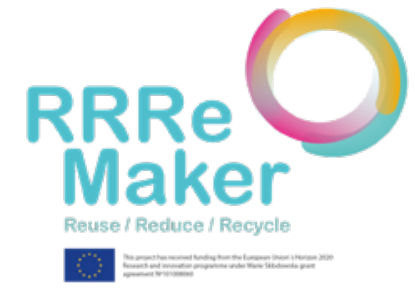

Proyecto Europeo de I+D+i "RRREMAKER. Reuse Reduce Recycle Al-based platform for automated and scalable Maker culture in Circular economy". Proyecto $\mathrm{H}$-2020 Marie Curie Research and Innovation Staff Mobility (H2O2OMarie SkXodowska-Curie Actions-RISE-2020, Ref. \#101008060), IP: Dra. Ana García López. Web: https://www.rrremaker.com 
Fig. 1. Diagrama diseñado por César González para RRREMAKER

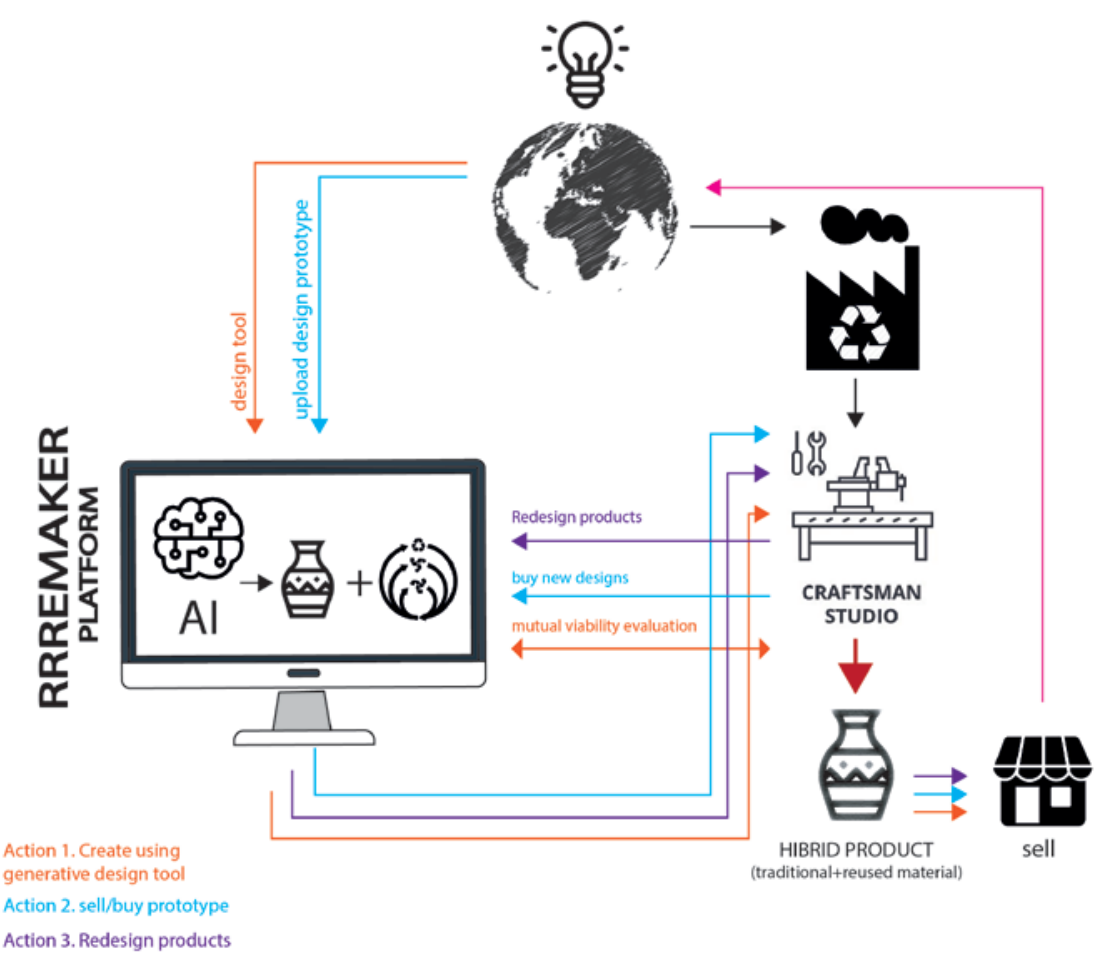

\section{Conclusiones}

En un entorno en que la digitalización, la creatividad, la innovación, la sostenibilidad y la tecnología han sido puestos en el foco de la cooperación entre artistas, ingenieros, arquitectos y diseñadores para la recuperación económica en las políticas más vanguardistas de la Europa post COVID, se hace especialmente interesante explorar los insumos que la investigación puede aportar a un sector tan trasversal como la artesanía desde el diseño y la aplicación de tecnologías disruptivas. La búsqueda de soluciones por parte de expertos en distintas áreas del conocimiento y la transferencia de ese conocimiento al pequeño y frágil ecosistema de talleres artesanos va a producir un impulso del sector que lo puede catapultar a la vanguardia de los procesos creativos en los que se puede apoyar la recuperación económica a partir de la crisis de la pandemia del 2020.

De entre esas áreas de conocimiento, el eco-diseño y la co-creación se postulan como ejes de la creatividad y la innovación pues el primero ataja los problemas medioambientales de raíz como factor adicional para la toma de decisiones (Hermida y Balboa, 2014:87). El ecodiseño aporta a la artesanía un papel estratégico al convertirse en motor de innovación y 
peldaño clave hacia la sostenibilidad y el consumo responsable ya que el $80 \%$ del impacto de un producto se determina justo en la fase de diseño ${ }^{18}$.

Dicho impulso, pasa indefectiblemente por la implementación de procesos complejos que aporta la tecnología (diseño generativo, fabricación digital, plataformas de co-creación, intercambio y transferencia de archivos a través de plataformas basadas en Inteligencia Artificial) pues serán, en un futuro cercano, herramientas digitales que orienten al artesano y le ayuden a simplificar algunos aspectos de su trabajo. Sin embargo, este viaje a la vanguardia no será completo si no se establece dentro parámetros de economía circular, si no se aborda, desde el propio proceso de diseño, el replanteamiento del objeto, sus procesos y su fabricación con nuevos materiales provenientes de materiales reciclados y recuperados. El artesano tradicional ya ha iniciado en muchos países su transformación al artesano maker, para compartir conocimientos, favoreciendo la colaboración en la producción de nuevos objetos, que sean más competitivos, creando oportunidades de emprendimiento y negocios colaborativos. Pero el cambio de paradigma completo a la economía circular es aún un tema por desarrollar.

18. Ver en la Guía de recomendaciones y buenas prácticas. Producción y consumo de productos artesanos sostenibles, publicado por el Instituto para el fomento del desarrollo y la formación. https://drive.google.com/ file/d/1OqfLwBEYnmM4ArQUoegnMMWgAXOOvdwD/view 


\section{Bibliografía y referencias}

ASEE. (2016). "Envisioning the Future of the Maker Movement: Summit Report". American Society for Engineering Education. Recuperado el 20/og/2021 de: https:/www.asee.org/documents/papers-and-publications/papers/ maker-summit-report.pdf

BRAUNGART, M.; MCDONOUGH, W.; BOLLINGER, A. (2007). "Cradle-to-cradle design: creating healthy emission- a strategy for eco-effective product and system design" en Journal of Cleaner production, Elsevier.

BRICEÑO GUTIÉRREZ, A. (2017). Escenarios distribuidos, albores de un nuevo modelo, Fundación DID, Chile. Recuperado el 23/09/2021en http://www.fundaciondid.cl/wp-content/uploads/2018/05/ escenarios_distribuidos_albores_AB.pdf

GONZÁLEZ MARTín, C. (2020). "Prefico co-, innovación abierta desde la colaboración", en García Lopez, A.y SUÁREZ MARTÍNEZ, A. (Coord.), Repensar la artesanía. Estrategias para impulsar la artesanía contemporánea. Comares, Granada.

GARCÍA LÓPEZ, A. (2020). "La investigación como estrategia para impulsar la artesanía" en García Lopez, A.y SUÁREZ MARTÍNEZ, A. (Coord.), Repensar la artesanía. Estrategias para impulsar la artesanía contemporánea. Comares, Granada.

GARCÍA LÓPEZ, A. (2021). "Nuevos paradigmas para la artesanía contemporánea en el contexto de la investigación en economía circular" en Meseguer Sánchez, et. Alt. (Ed.), Economía circular: fundamentos y aplicaciones, Thomson Reuters Aranzadi, Pamplona.

HATCH, M. (2014). The Maker Movement Manifesto. DOI: 10.1162/INOV_a_00135

HeRmidA BALBOA, C.; Dominguez Somonte, M. (2014). Economía circular como marco para el ecodiseño: el modelo ECO-3. Recuperado el 10/o9/2021 de: https://www2.uned.es/egi/publicaciones/articulos/Economia_circular_ como_marco_para_el_ecodiseno_el_modelo_ECO-3.pdf

MALAKUCZI, V. (2019). Computational by design, Common Ground Research Networs, University of Illinois Research Park, Campaign, IL. ISBN 9781863351218

MARTín, J. (2017). La era del prosumidor. Recuperado el 02/08/2021 de Cerem: https://www.cerem.es/blog/ la-era-del-prosumidor

RitZer, G., JURGEnSON, N. (2010). "Production, Consumption, Prosumption. The nature of capitalism in the age of the digital 'prosumer'". Journal of Consumer Culture, 10(1), 13-36. DOI: 10.1177/1469540509354673

VVAA_Guía de recomendaciones y buenas prácticas. Producción y consumo de productos artesanos sostenibles, publicado por el Instituto para el fomento del desarrollo y la formación. Recuperado el 24/09/2021 de: https:// drive.google.com/file/d/1OqfLwBEYnmM4ArQUoegnMMWgAXOOvdwD/view

VVAA_Report on the situation of craftsmanship in Europe.CR@FTSMAN PROJECTES/og/LLP-LdV/ TOI/149072. Recuperado el 24/09/2020 de: http://projects.ifes.es/pdfs/craft/craftı.pdf

VVAA_II Plan integral para el fomento de la artesanía en Andalucía (2014-2017). Recuperado el 12/09/2021 de: http://www.juntadeandalucia.es/turismoycomercio/publicaciones/14347486o.pdf 